536. 33 : 662. 612. 32

\title{
A Study on Radiation from a Luminous Flame*
}

\author{
By Yasuo MoRI** and Keiji MAKINo***
}

From such a standpoint that a radiation from a luminous flame is due to the small solid particles (so-called "soot"), a fundamental research for the radiation from the luminous flames of the propane-air and city gas-air was carried out on the basis of Mie theory concerning radiation.

The purpose of this paper is to measure the scattered parts and attenuated parts of incident lights about a flame and study the radiation of a luminous flame systematically.

A mean diameter $D_{32}$ of soot particles, defined as $D_{32}=\int_{0}^{D_{\infty}} N(D) D^{3} d D / \int_{0}^{D_{\infty}} N(D) D^{2} d D$, where $N(D) d D$ is the probability that the soot diameter is in the range of $D$ and $D+d D$, is suggested to discuss the relation between the scattered light from the flame and the attenuated light by the luminous flame.

It is showed that the values of $D_{32}$ obtained by scattering and attenuation are $1400 \AA$ regard. less of diffusion or premixed flame.

The density of the soot particles, $C_{v}$, is experimentally obtained from the attenuation of the incident light in our research, and general consideration of radiation from luminous flames is given.

\section{Introduction}

Radiation from a strong orange-yellow colored flame, i.e., a luminous flame, obtained in combustion of hydrocarbon fuel, is regarded as radiation from solid particles at high temperatures in a luminous flame. However, according to a recent paper by Echigo et al. (1) on the measurement of spectrum analysis of a Juminous flame, it was reported that the radiation from a luminous flame is not considered as a radiation from solid particles, because its spectrum is not continuous, but the paper's conclusion is not so decisive as to deny the existence of solid particles. One of the methods to examine the existence of solid particles is to make a measurement of scatter phenomena of particles by sending a light beam through a flame from outside. In this study, we will examine the existence of solid particles in several kinds of flames by means of a scattered light. As mentioned below, some kinds of luminous flames have considerable brightness without the existence of solid particles, and the paper by Echigo et al. is considered to be concerned with these flames.

Therefore, in this study, we regarded materials

* Received 6th July, 1968.

** Prolessor, Faculty of Engineering, Tokyo Institute of Technology.

*** Graduate Course Student, Faculty of Engineering, Tokyo Institute of Technology, Meguro-ku, Tokyo. in a luminous flame as solid particles and obtained a mean diameter of soot particles in a luminous. flame. Many investigations concerning the relation between the soot concentration and the emissivity have been done, but, in most cases, soot particles. are considered to have the same diameter. However, the diameter of soot particles is not always. the same, as seen in pictures of soots taken through an electron microscope. Therefore, we assumed, in our study, that the diameter of soot particles had a distribution, and obtained a mean diameter of soot. particles by means of a light-scattering technique, by the use of the well-known Mie's theory on the scatter and absorption of a light beam by a sphere. The diameter of soot ${ }^{(2)}(3)$ and emission ${ }^{(4)}$ from it have been measured, but so far no systematic in. vestigation on radiation from a luminous flame containing solid particles has been done, in which both measurements of soot diameter and emissivity are made at the same time for one luminous flame and. the relation between them is discussed. The object of this study is: to measure the diameter of soot in luminous flames of some kinds of gaseous fuel by light scattering; to measure the decrement of a transmitted light through a luminous flame by use of two different wavelengths instead of measuring the emissivity; to obtain the diameter of soot from. those results and compare it with that obtained by a light-scattering technique to verify Mie's theory' 
experimentally; and, to collect basic data for radiation from a luminous flame containing small solid particles. On the assumption that the absorption factor is equal to the emissivity, instead of making measurement of the emissivity of a luminous flame, the decrement of a transmitted light is measured in this report. Therefore, when the mean diameters obtained by these two methods agree with each other, Mie's theory will be experimentally verified. That is, the emissivity of a luminous flame is theoretically calculable from the mean diameter obtained by a light-scattering technique. We used pure propane gas and city gas as fuel.

\section{Nomenclature}

$C_{n}$ : number density of soot $\mathrm{cm}^{-3}$

$C_{v}$ : volumetric density of soot $\mathrm{cm}^{3} / \mathrm{cm}^{3}$

$D$ : diameter of soot particle $\AA$

$D_{32}$ : mean diameter of soot particle $\AA$

$I$ : light intensity $\mathrm{w} / \mathrm{cm}^{2}$

$N(D)$ : probability of soot diameter being $D$

$Q_{\text {ext }}$ : effective factor

$\bar{Q}_{\text {est }}:$ mean effective factor

$S(\theta)$ : amplitude function

$\overline{|S(\theta)|^{2}}$ : mean amplitude function

$k=2 \pi / \lambda$

$l$ : optical path $\mathrm{m}$

$m$ : complex refractive index of soot $=n-i k$

$r$ : distance from a sphere $\mathrm{m}$

$x=\pi D / \lambda$

$\Delta V$ : elementary volume of luminous flame observed $\mathrm{m}^{3}$

$\lambda$ : wavelength $\AA$

$\tau:$ transmittency

Subscript

0 : incident light

1: polarized light whose vibrating plane is perpendicular to the plane containing incident and scattered lights

2: polarized light whose vibrating plane is parallel to the previous plane

$\infty$ : maximum diameter

\section{Theoretical analysis}

2.1 Determination of the diameter of a soot particle by a light-scattering technique

When the light beam of the wavelength $\lambda$ and

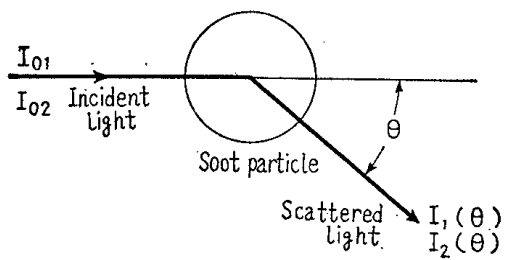

Fig. 1 Light-scattering by a particle the intensity $I_{0}$ is incident on a soot particle of the diameter $D$, as shown in Fig. 1, the intensity of a light scattered toward $\theta$ in the figure is given as follows, according to the Mie's theory ${ }^{(5)}(6)$ :

$$
I_{1}(\theta)=\frac{\left|S_{1}(\theta)\right|^{2}}{k^{2} r^{2}} I_{01}, I_{2}(\theta)=\frac{\left|S_{2}(\theta)\right|^{2}}{k^{2} r^{2}} I_{02}
$$

where $r$ is the distance from the particle. $I_{01}$ is the intensity of the polarized light, whose vibrating plane is perpendicular to the plane (in Fig. 1) containing incident and scattered lights, of the incident light, and $I_{02}$ is that of the polarized light whose vibrating plane is parallel to the plane containing incident and scattered lights. $\left|S_{1}(\theta)\right|^{2}$ and $\left|S_{2}(\theta)\right|^{2}$ are functions of $x=\pi D / \lambda$, complex refractive: index of $m$, and $\theta$ and are written as :

$$
\left.\begin{array}{l}
\left|S_{1}(\theta)\right|^{2}=\mid \sum_{n=1}^{\infty} \frac{2 n+1}{n(n+1)} \\
\quad \times\left.\left\{a_{n} \pi_{n}(\cos \theta)+b_{n} \tau_{n}(\cos \theta)\right\}\right|^{2} \\
\left|S_{2}(\theta)\right|^{2}=\mid \sum_{n=1}^{\infty} \frac{2 n+1}{n(n+1)} \\
\quad \times\left.\left\{b_{n} \pi_{n}(\cos \theta)+a_{n} \tau_{n}(\cos \theta)\right\}\right|^{2}
\end{array}\right\}
$$

here

$$
\left.\begin{array}{l}
\pi_{n}(\cos \theta)=\frac{P_{n}^{1}(\cos \theta)}{\sin \theta} \\
\tau_{n}(\cos \theta)=\frac{d}{d \theta} P_{n}^{1}(\cos \theta)
\end{array}\right\}
$$

where $P_{n}{ }^{1}(\cos \theta)$ is a Legendre function. However.

$$
\left.\begin{array}{l}
a_{n}=\frac{2^{n} x^{n+1}(n+1) n !}{(2 n+1) !} \frac{Q_{n}{ }^{\prime} P_{n}-m^{2} Q_{n} P_{n}^{\prime}}{(n+1) Q_{n}{ }^{\prime} \zeta_{n}-m^{2} x Q_{n} \zeta_{n}{ }^{\prime}} \\
b_{n}=\frac{2^{n} x^{n+1}(n+1) n !}{(2 n+1) !} \frac{Q_{n}{ }^{\prime} P_{n}-Q_{n} P_{n}^{\prime}}{(n+1) Q_{n}^{\prime} \zeta_{n}-x Q_{n} \zeta_{n}{ }^{\prime}}
\end{array}\right\}
$$

$Q_{n}, Q_{n}{ }^{\prime}, P_{n}, Q_{n}^{\prime}$ are expressed as follows :

$$
\begin{aligned}
& Q_{n}=1+\sum_{j=1}^{\infty} \prod_{r=1}^{j}\left\{\frac{\left(-m^{2} x^{2}\right)}{2 r(2 n+2 r+1)}\right\} \quad \ldots \ldots \ldots \ldots \ldots \\
& Q_{n}{ }^{\prime}=1+\sum_{j=1}^{\infty}\left[\frac{2 j+n+1}{n+1} \prod_{r=1}^{j}\left\{\frac{\left(-m^{2} x^{2}\right)}{2 r(2 n+2 r+1)}\right\}\right] \\
& P_{n}=1+\sum_{j=1}^{\infty} \prod_{r=1}^{j}\left\{\frac{\left(-x^{2}\right)}{2 r(2 n+2 r+1)}\right\} \ldots \ldots \ldots \ldots \ldots . . . \cdots \\
& P_{n}{ }^{\prime}=1+\sum_{j=1}^{\infty}\left[\frac{2 j+n+1}{n+1} \prod_{r=1}^{j}\left\{\frac{\left(-x^{2}\right)}{2 r(2 n+2 r+1)}\right\}\right]
\end{aligned}
$$

Moreover

$$
\left.\begin{array}{l}
\zeta_{n}(x)=x h_{n}{ }^{(2)}(x) \\
\zeta_{n}{ }^{\prime \prime}(x)=h_{n}{ }^{(2)}(x)+x h_{n}{ }^{(2)}{ }^{\prime}(x)
\end{array}\right\}
$$

Next, $h_{n}{ }^{(2)}(x)$, and $h_{n}{ }^{(2)}(x)$ are expressed by the following equations :

$$
\left.\begin{array}{l}
h_{n}{ }^{(2)}(x)=\frac{2 n-1}{x} h_{n-1}{ }^{(2)}(x)-h_{n-2}{ }^{(2)}(x) \\
h_{n}^{(2) \prime}(x)=h_{n-1}{ }^{(2)}(x)-\frac{n+1}{x} h_{n}{ }^{(2)}(x)
\end{array}\right\}
$$


We can calculate $\left|S_{1}(\theta)\right|^{2}$ and $\left|S_{2}(\theta)\right|^{2}$ from these equations. These equations are numerically computed, by the use of a FACOM 222, assuming $\theta$ to be $50^{\circ}$.

The value of the complex refractive index of soot was uncertain and was taken as a parameter in this calculation. The experimental values are given by Senftleben-Benedict ${ }^{(7)}$ for carbon, by Thring ${ }^{(8)}$ and others for pygrographite, and by Mori et al. (9) for carbon: - Recently, Dalzall-Sarofim ${ }^{(10)}$ measured about pressed test pieces of soot.

No great difference is seen between them, but we adopted the value by Senftleben et al. by reason mentioned below. This value is shown below;

$\begin{array}{rcc}\text { Wavelength } \AA & n & k \\ 4360 & 1.90 & 0.68 \\ 4920 & 1.94 & 0.66 \\ 5460 & 1.96 & 0.66 \\ 5780 & 1.97 & 0.65 \\ 6230 & 2.00 & 0.66\end{array}$

The wavelength of $4500 \AA$ in a light-scattering technique and those of $4500 \AA, 6000 \AA$ and $7300 \AA$ are used in the measurement of transmittency. The complex refractive indices of soot for the wavelengths of $4500 \AA$ and $6000 \AA$ were interpolated and obtained as follows:

Wavelength $4500 \AA \quad m=1.91-0.675 i$

Wavelength $6000 \AA \quad m=1.99-0.655 i$

The value of $m$ at the wavelength of $7300 \AA$ was so taken as to agree with the other experimental results, as mentioned below.

We will discuss a case in which the diameter of soot particles has a distribution. We assume the probability of a diameter of $D$ to be $N(D)$ and the diameter of soot particles in the luminous flame to be continuously distributed from 0 to $D_{\infty} . \quad N(D)$ is expressed from the definition as:

$$
\int_{0}^{D_{\infty}} N(D) d D=1
$$

Since the density of soot is very small in an actual luminous flame, the intensity of a scattered light is considered to be proportional to the number of soot particles. Consider a flame of the volume of $\Delta V$, and suppose $C_{n}$ to be the number density of soot, then from Eq. (1) we have

$$
\left.\begin{array}{l}
I_{1}(\theta)=\int_{0}^{D_{\infty}}\left|S_{1}(\theta)\right|^{2} I_{01} C_{n} N(D) \Delta V /\left(k^{2} r^{2}\right) d D \\
I_{2}(\theta)=\int_{0}^{D_{\infty}}\left|S_{2}(\theta)\right|^{2} I_{02} C_{n} N(D) \Delta V /\left(k^{2} r^{2}\right) d D
\end{array}\right\}
$$

The mean diameter $D_{32}$ of soot particles is defined as ${ }^{(11)}$ :

$$
D_{32} \equiv \int_{0}^{D_{\infty}} N(D) D^{3} d D / \int_{0}^{D_{\infty}} N(D) D^{2} d D
$$

In order to make the significance of $D_{32}$ clear, a ratio of the total volume of particles to their total surface area in a flame is written as follows:

$$
\begin{aligned}
& \frac{\int_{0}^{D_{\infty}} C_{n} N(D) \frac{4}{3} \pi D^{3} \frac{1}{8} d D}{\int_{0}^{D_{\infty}} C_{n} N(D) 4 \pi D^{2} \frac{1}{4} d D}=\frac{\int_{0}^{D_{\infty}} N(D) D^{3} d D}{6 \int_{0}^{D_{\infty}} N(D) D^{2} d D} \\
& =\frac{D_{32}}{6} \text {. }
\end{aligned}
$$

The following are defined as:

$$
\left.\begin{array}{c}
\overline{\left|S_{1}(\theta)\right|^{2}} \equiv \frac{\int_{0}^{D_{\infty}}\left|S_{1}(\theta)\right|^{2} N(D) d D}{\int_{0}^{D_{\infty}} N(D) D^{2} d D} \\
\overline{\left|S_{2}(\theta)\right|^{2}} \equiv \frac{\int_{0}^{D_{\infty}}\left|S_{2}(\theta)\right|^{2} N(D) d D}{\int_{0}^{D_{\infty}} N(D) D^{2} d D}
\end{array}\right\}
$$

and

$$
C_{v}=C_{n} \frac{\pi}{6} \int_{0}^{D_{\infty}} N(D) D^{3} d D
$$

Then, substituting Eqs. (15), (17), and (18) into Eq. (14), we have

$$
\left.\begin{array}{l}
I_{1}(\theta)=\frac{6 C_{v} \mid \overline{\left.S_{1}(\theta)\right|^{2}}}{\pi k^{2} r^{2} D_{32}} \Delta V I_{01} \\
I_{2}(\theta)=\frac{6 C_{v}\left|\overline{S_{2}(\theta)}\right|^{2}}{\pi k^{2} r^{2} D_{32}} \Delta V I_{02}
\end{array}\right\}
$$

From Eq. (19), we have the following equation:

$$
\frac{I_{1}(\theta)}{I_{2}(\theta)}=\frac{\overline{\left|S_{1}(\theta)\right|^{2}}}{\mid \overline{\left.S_{2}(\theta)\right|^{2}}}
$$

The introduction of this newly-defined $D_{32}$ reduces to such a simple equation of a clear physical significance as Eq. (20) even when the diameter of soot particles has a distribution.

We consider the following two distributions as examples of distributions of the diameter of soot particles.

$$
\begin{aligned}
& N(D) \sim D / D_{\infty}-\left(D / D_{\infty}\right)^{2} \\
& N(D) \sim \frac{\exp \left\{-\left[1.26 \ln 1.13 D /\left(D_{\infty}-D\right)\right]^{2}\right\}}{D^{4}\left(D_{\infty}-D\right)}
\end{aligned}
$$

They are shown in Fig. 2.

Equation (21) indicates a parabola curve, and

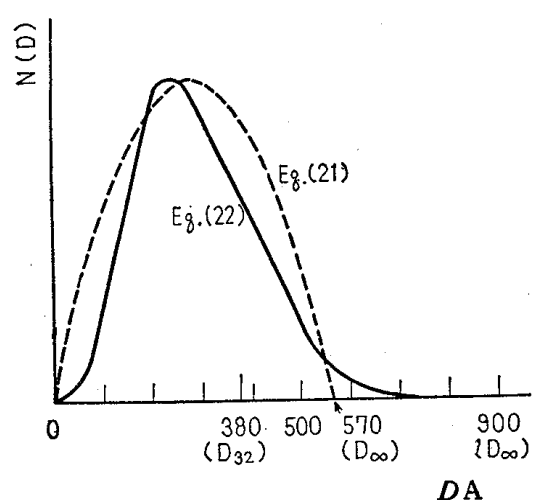

Fig. 2 Diameter distribution of soot particles 
Eq. (22) represents the distribution (11) of the diameter of polystyrene small particles formed by condensation, and we adopt this distribution, as we think that soot within a luminous flame is formed by condensation. Two distributions in Fig. 2 have the same $D_{32}(380 \AA) . \quad D_{\infty}$ in Eq. (21) is $570 \AA$ and that in Eq. (22) is $900 \AA$. The right side of Eq. (20) can be computed for a given distribution and is computed by use of an electronic computer, assuming $\theta$ to be $50^{\circ}$ as before. The right side of Eq. (20) for $D_{32}$ is shown in Fig. 3 for two distributions of Eqs. (21) and (22), and the uniform diameter. We find from Fig. 3 that the curves nearly agree with each other for two different distributions and also for a uniform diameter. Namely, the right side of Eq. (20) is determined only by $D_{32}$, and it hardly depends upon the distribution of the diameter of the soot particle. Therefore, in this study, we adopt only the distribution of Eq. (22). As we measure a value on the ordinate, we can determine $D_{32}$ in a way illustrated in the figure.

2.2 Determination of a diameter of a soot particle by the measurement of a transmittency

When a light beam is sent to soot particles which have the same diameter in a light beam, the ratio $\tau$ of the intensities of incident to transmitted lights is expressed as :

$$
\tau=\frac{I}{I_{0}}=\exp \left[-\frac{\pi D^{2} C_{n} l}{4} Q_{\text {ext }}\right]
$$

where $Q_{\text {ext }}$ is the value computed from Mie's theory, which is given below:

$$
Q_{\text {ext }}=\frac{2}{x^{2}} \sum_{n=1}^{\infty}(2 n+1) R_{e}\left(a_{n}+b_{n}\right)
$$

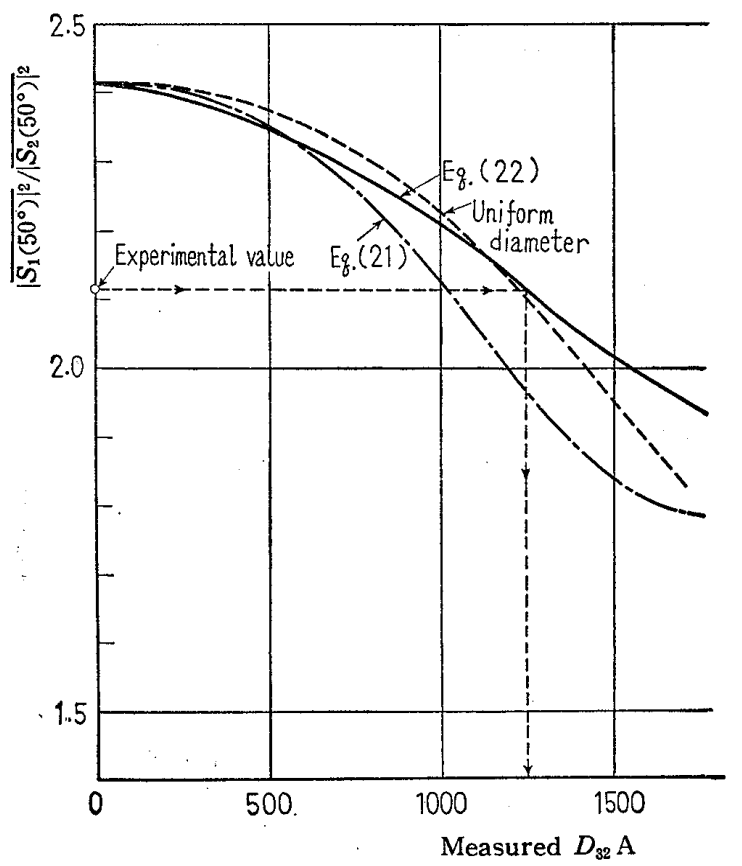

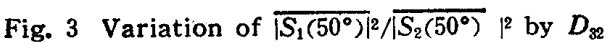

$a_{n}$, and $b_{n}$ being derived from Eq. (4), and $R_{e}$ ( ) representing the real part of $\left(\right.$ ). $Q_{\text {ext. }}$ is computable for given $x$ and $m$. We used three wavelengths of $4500 \AA, 6000 \AA$ and $7300 \AA$ in the measurement of the diameter of a soot particle from. the transmittency. Values of $m$ at wavelengths of $4500 \AA$ and $6000 \AA$ are derived from Eqs. (11) and (12), and that at the wavelength of $7300 \AA$ is determined as follows, so that it agrees with the other experimental value.

$$
m=2.07-0.8 i
$$

We will discuss a case when the diameter of a soot particle has a distribution as discussed in 2.1 . We can express Eq. (23) as :

$$
\tau=\exp \left[-\frac{\pi C_{n} l}{4} \int_{0}^{D_{\infty}} Q_{\text {ext }} N(D) D^{2} d D\right]
$$

where the diameter of the soot particles varies from 0 to $D_{\infty}$. The definition of a mean effective factor is :

$$
\bar{Q}_{\mathrm{ext}} \equiv \frac{\int_{0}^{D_{\infty}} Q_{\mathrm{ext}} N(D) D^{2} d D}{\int_{0}^{D_{\infty}} N(D) D^{2} d D}
$$

We write Eq. (26) by the use of Eqs. (15), (18) and (27) as follows :

$$
\tau=\exp \left[-\frac{\overline{3 Q}_{\mathrm{ext}} C_{v} l}{2 D_{32}}\right]
$$

where $l$ is already known, but $D_{32}$ and $C_{v}$ of soot particles within a luminous flame are not generally known. When either of them is known, the other can be obtained by measuring the transmittency $\tau$. Therefore, we tried to take away the influence of density, that is, to take logarithmic values of both sides of Eq. (28).

$$
\ln \tau^{-1}=\frac{3 \bar{Q}_{e x t} C_{v} l}{2 D_{32}}
$$

Equation (29) is effective for two different wavelengths of $\lambda_{1}$ and $\lambda_{2}$ as :

$$
\left.\begin{array}{l}
\ln \tau^{-1}\left(\lambda_{1}\right)=\frac{3 \bar{Q}_{\text {ext }}\left(\lambda_{1}\right) C_{v} l}{2 D_{32}} \\
\ln \tau^{-1}\left(\lambda_{2}\right)=\frac{3 \bar{Q}_{\text {ext }}\left(\lambda_{2}\right) C_{v} l}{2 D_{32}}
\end{array}\right\}
$$

Therefore we obtain

$$
\frac{\ln \tau^{-1}\left(\lambda_{1}\right)}{\ln \tau^{-1}\left(\lambda_{2}\right)}=\frac{\bar{Q}_{\mathrm{ext}}\left(\lambda_{1}\right)}{\bar{Q}_{\mathrm{ext}}\left(\lambda_{2}\right)}
$$

The right side of Eq. (31) can be calculated for the fixed wavelengths of $\lambda_{1}$ and $\lambda_{2}$. Assuming $\lambda_{1}$ to be $4500 \AA$, and $\lambda_{2}$ to be $6000 \AA$ and $7300 \AA$, we com. puted Eq. (27) by use of $Q_{\text {ext }}(4500), Q_{\text {exi }}(6000)$ and $Q_{\text {ext }}(7300)$ given by Eq. (24), by means of an electronic computer. The solution of the right side of Eq. (31) is illustrated in Fig. 4. $N(D)$ is calculated and shown for both Eqs. (21) and (22), and also for the case of the uniform diameter. $\bar{Q}_{\text {ext }}$ (4500) $\bar{Q}_{\text {ext }}(7300)$ is only shown for the case of 
Eq. (22). Concerning the curve of $\bar{Q}_{\text {ext }}(4.500) / \bar{Q}_{\text {ext }}$ $(6000)$ in the figure, considerable agreement is found between Eqs. (21) and (22), in spite of different distributions. Namely, they only depend upon $D_{32}$, and they are hardly influenced by their distributions. In this study, we adopted only the diameter distribution for condensation, i.e., Eq. (22). We can obtain $D_{32}$ following the route indicated by an arrow from a measured value on the ordinate in the figure.

\section{Experimental apparatus}

The optical system is drawn in Fig. 5. A xenon short arc lamp was used as a light source, and an incident light beam was chopped at the frequency of $1250 \mathrm{c} / \mathrm{sec}$. In this way we selected the light beam of $1250 \mathrm{c} / \mathrm{sec}$ coming from a luminous flame and detected it. The d.c. part of radiant light obtained directly from the luminous flame is not led to the amplifier, but only the scattered light in the a.c. part is amplified. We can cobtain the intensity of the scattered light at the routput. We set interference filters to detect a single

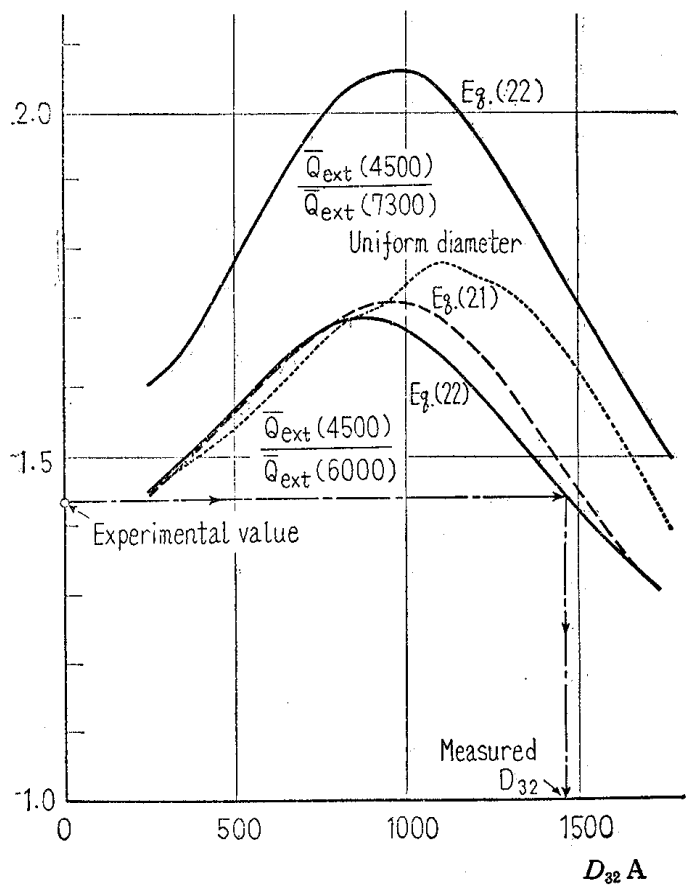

Fig. 4 Variation of $\bar{Q}_{\text {ext }}(4500) / \bar{Q}_{\text {ext }}(6000)$ and $\bar{Q}_{\text {ext }}(4500) / \bar{Q}_{\text {ext }}(7300)$ by $D_{32}$



Fig. 5 Optical apparatus wavelength and a polarizer to vary a polarized plane, in front of a detector. We used three interference filters, having the central wavelengths of $4500 \AA, 6000 \AA$ and $7300 \AA$. With them we also used colored glass filters except for $4500 \AA$, to eliminate a subsidiary transmitted band. The interference filters, the polarizer and the photomultiplier are set together on the optical bench and the bench rotates around a burner in the range of $\theta=$ $0 \sim 140^{\circ}$ or so. We measured a transmitted light at the angle of $\theta=0^{\circ}$, and a scattered light at $\theta=50^{\circ}$.

Mostly pure propane gas was used in the experiment and some city gas was used, too. The structure of the burner and the flow sheet of fuel system are shown in Fig. 6. We used a diffusion flame and the inner diameter of a burner port was $7 \mathrm{~mm}$. A small amount of air was introduced around the burner port to maintain a stable flame. We can mix oxygen with the air current to examine the influence of temperature of flame upon the complex refractive index of soot. We also introduce the air into a fuel tube for experiments of an airpremixed flame. We can vary the height of the flame by regulating the fuel flow rate by a valve. An optical bench can be moved up and down along the axis of the flame in order to measure various points along the axis of flame. We measured the temperature of the flame with a $0.1 \mathrm{~mm} \mathrm{pt-pt} \mathrm{Rh}$ thermo-couple. No correction has been made on the radiation and conduction error for the thermo-couple

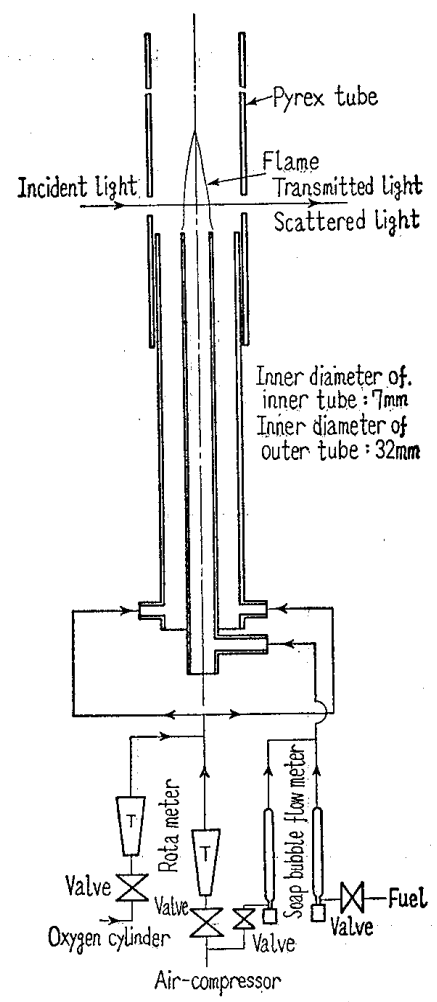

Fig. 6 Burner 
measurement. We measured the thickness of flame l. from photos.

\section{Experimental verification of Mie's theory}

Comparison between mean diameters of $D_{32}$, obtained from the measurement of the intensities of transmitted and light-scattered lights, is illustrated in Fig. 7. We used diffusion flames of pure propane gas and made experiments on luminous flames up to the height of about $50 \mathrm{~mm}$. The abscissa represents a position of a flame with the origin taken at the top of a burner port. We find good agreement between the values of $D_{32}$ obtained by three methods. $D_{32}$ thus obtained is about $1400 \AA$ and remains the same in the direction of flame height. It can be explained as follows: soot particles will burn as they move upwards in the flame. However, only the soot particles in the very thin part near the outer surface of the flame will instantaneously react with oxygen in the air, and those in the central part of the flame will have no reaction. We can presume this by observation of the flame. The condition of the flame surface is different from that of the central part. The former is much more transparent than the latter. Soot particles burn and disappear near the surface, and most particles in the central part neither grow nor decrease. It is considered from these facts that a mean diameter of $D_{32}$ of soot particles is almost constant in the direction of the flame height. Jinno et al. (12) made experiments on a wider part of a flame on the assumption that the diameter of soot particles is uniform. According to their report, the diameter has a tendency to decrease in the direction of the flame height, but it is almost constant in a range from $50 \%$ to $80 \%$ of flame height. The range of our measurement lies from $60 \%$ to $80 \%$ of flame height, and this agrees with the tendency reported by Jinno et al.

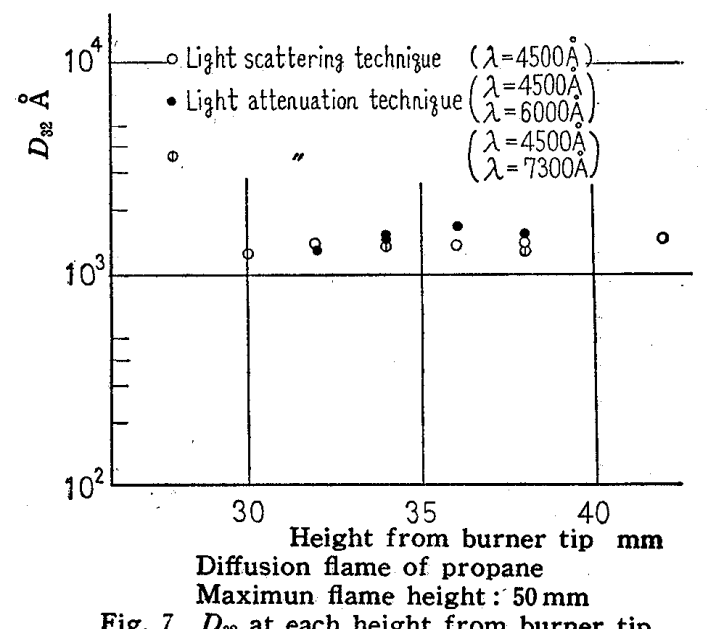

The diameter of soot particles measured from photos of soot particles collected on a glass, taken through an electron microscope, is approximately $450 \AA$, which is fairly different from the value of $D_{32}=1400 \AA$ obtained in this study. It is considered that soot particles on a glass grow to a large particle when they are cooled and agglomerated. It is known that diameters of some large soot particles reach several thousand $\AA$, but it is doubtful that the size and distribution of soot particles collected on a glass are the same as those of soot particles in a flame. It is also not clear that the physical nature of a soot particle in a luminous flame is the same as that on a glass. Hottel et al. (13) assumed that soot particles had a uniform diameter and simply compared a geometrically mean diameter of soot particles, obtained by means of a light-scattering technique, with that of soot particles collected on a glass at room temperature. According to their report, a mean diameter of the former is about $1400 \AA$ and the latter about $250 \AA$. A considerable difference is seen between them. They made experiments on the scatter of light by particles which consist mainly of those of the diameter of $250 \AA$ and large particles of the diameter of $1850 \mathrm{~A}$ and of the number density of $2 \times 10^{-5}$, and reported that the scattered light in this case is the same as that for a uniform diameter of $1400 \AA$. From this, they reported that large particles of small number density in a flame or agglomerated lumps of soot particles have a great influence upon the measurement. However, the problem in their study lies in the method of collection of soot particles, and the assumption that the two specific sizes of soot particles are dominant is doubtful from a stand point of mechanism of soot generation. We estimate a continuous distribution of the diameter of soot particles in this study; furthermore, we suggest a representative diameter for convenience of calculation of emissivity. Therefore, $D_{32}$ obtained in this study may be considered as an effective mean diameter of soot particles in a flame. There is no necessity to require an agreement between $D_{32}$ and a diameter obtained from photos through an electron microscope. We regard that Mie's theory is verified by the use of this effective diameter. It can be said that when we use this effective diameter, the intensities of both transmitted and scattered lights by soot particles in a luminous flame experimentally agree with the values calculated from Mie's theory. It is possible to obtain $D_{32}$ from a light-scattering experiment at one wavelength and compute $C_{v}$ from Eq. (27) with the data of transmittency measured at this wavelength. Since $C_{v}$ and $D_{32}$ thus obtained are peculiar to the flame and independent of a wavelength, an emissivity of a luminous flame at other wavelengths 
can be obtained from Mie's theory, and it may be concluded that it agrees with an experimental value.

We adopt the visible region in this study, but we should call attention to this method when it is applied to the infrared region which is important in practical cases. Namely, since the values of the physical properties of soot by Senftleben-Benedict (7), which are used in this study, are only reliable in the visible region, we have to use other data for the infrared region.

In the case of a combustion of liquid fuel, we have to consider another effective diameter, as large particles in a flame of this kind of combustion have a diameter of more than $1 \mu$ (that is, in a case of $x \geqq 1$ ). However, we will reserve it for another occasion.

We considered a distribution of the diameter of soot particles in this study, but, without considering a distribution, poor agreement is found between a diameter of particles measured by a light-scattering technique and that by an attenuation method. The curve for uniform diameter in Fig. 3 is almost the same as those having distributions concerning a light-scattering technique. However, the curve of a uniform diameter in Fig. 4 concerning an attenuation method fairly differs from those having distributions in the range above $1000 \AA$. A diameter, estimated to be uniform and obtained by a lightscattering technique, is about $1300 \AA$, and that obtained from a transmittency is about $1700 \AA$. We find poor agreement between them. An emissivity of a luminous flame computed from Mie's theory on the assumption of uniform diameter does not generally agree with experimental values.

If the physical properties of $n$ and $k$ of soot particles at an arbitrary wavelength are known, $Q_{\text {ext }}$ is obtained from Mie's theory. $\bar{Q}_{\text {ext }}$ at a wavelength is computed from Eq. (27) by the use of $D_{32}$ obtained from experiments in which, for example, wavelengths of $4500 \AA, 6000 \AA$ and $7300 \AA$ are used. A transmittency is calculable from Eq. (28) by the use of $C_{v}$ obtained from experiments. For a luminous flame in a state of thermal equilibrium, an absorbtivity may be considered to be equal to an emissivity as mentioned above, and an emissivity at an arbitrary wavelength is obtained.

From $D_{32}$ obtained in our experiments and given $C_{v}$, we get a transmittency at an arbitrary wavelength. After we make integral calculus of them over all wavelengths and find the average, we can compute the total emissivity of a luminous flame. So far, it is well known how to calculate an emissivity from a geometrical mean diameter and the approximate values of $n$ and $k$. A mean diameter of $D_{32}$ to be reasonably used for a calculation of an emissivity of soot particles was obtained in this study. However, the values of $n$ and $k$ of soot particles for all wavelengths at temperatures of luminous flame are uncertain, and even though $D_{32}$ is obtained in our experiments, an exact emissivity of soot particles in a luminous flame will not be obtained from it. An exact emissivity and the comparison between theory and experiment for it are expected from future studies.

\section{Experiment by diffusion flame}

Oxygen was added in the flame stabilizing air flow to measure a flame at various temperatures. The flame height becomes shorter with an increase in the flow rate of oxygen. Two cases of experiments were performed. First, the flow rate of fuel was increased and the flame height was kept constant at $50 \mathrm{~mm}$; and second, the flow rate was held constant and the flame height was changed.

5.1 A case in which propane was used as fuel

5.1.1 Experiment on the constant flame height $(50 \mathrm{~mm})$

(a) Variation of $D_{32}$ :

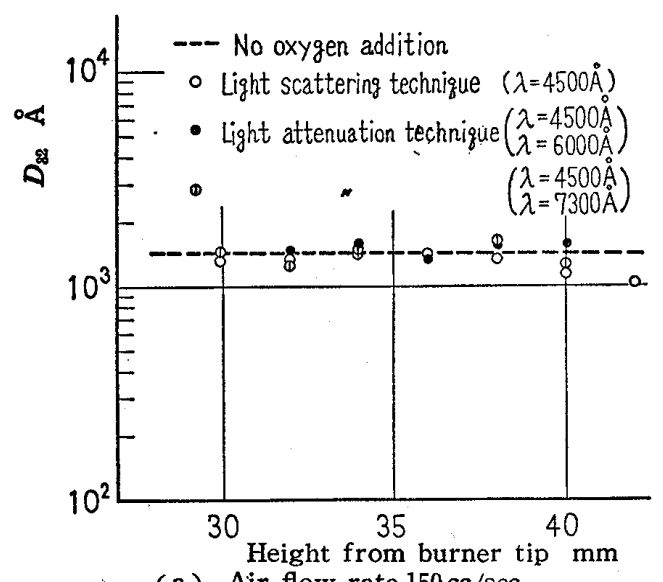

(a) Air flow rate $150 \mathrm{cc} / \mathrm{sec}$

Oxygen flow rate $22 \mathrm{cc} / \mathrm{sec}$

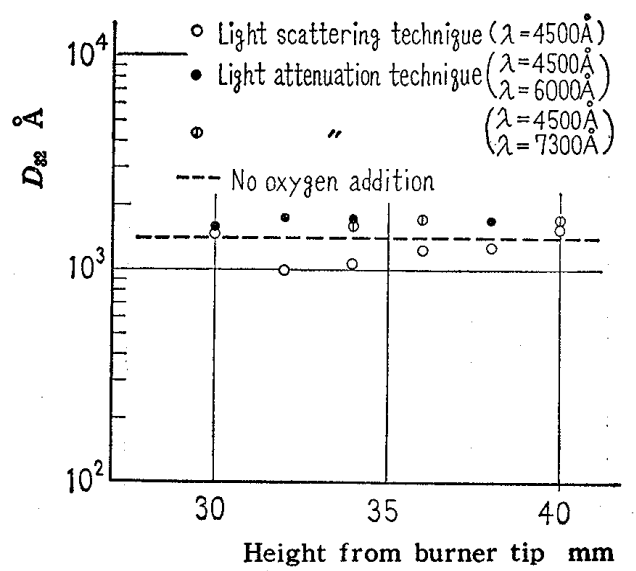

(b) Air flow rate $150 \mathrm{cc} / \mathrm{sec}$ Oxygen flow rate $80 \mathrm{cc} / \mathrm{sec}$

Fig. 8 Diffusion flame of propane 
The comparison between values of $D_{32}$ for two kinds of flow rate of oxygen measured by means of three methods are illustrated in Fig. 8 (a) and (b). When the flame temperature is not high at a small flow rate of oxygen, good agreement is found between these values of $D_{32}$. They are about $1400 \AA$, which are the same as those in Fig. 7. They have no change in the direction of flame height. It is explained in the same way as in 4. However, when the flame temperature is raised with an in. crease of flow rate of oxygen, the value of $D_{32}$ obtained by use of a scattered light becomes small,

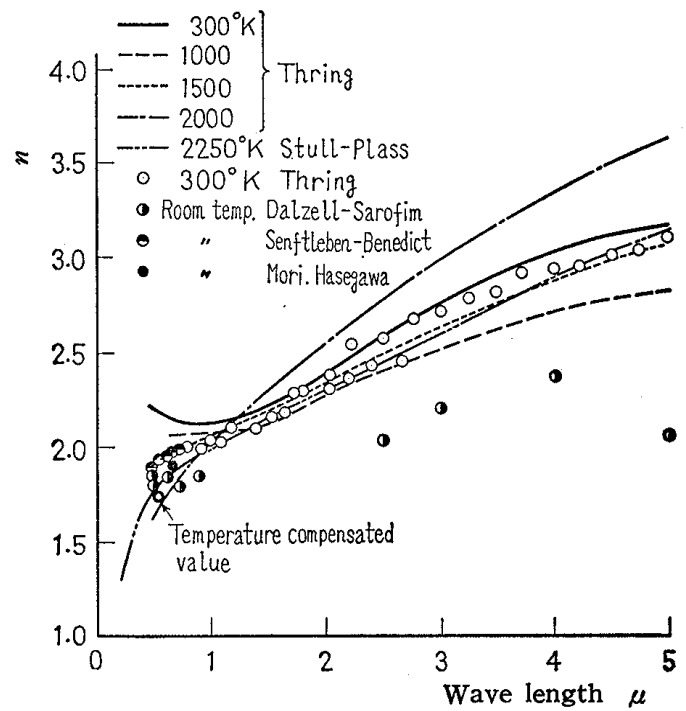

(a)

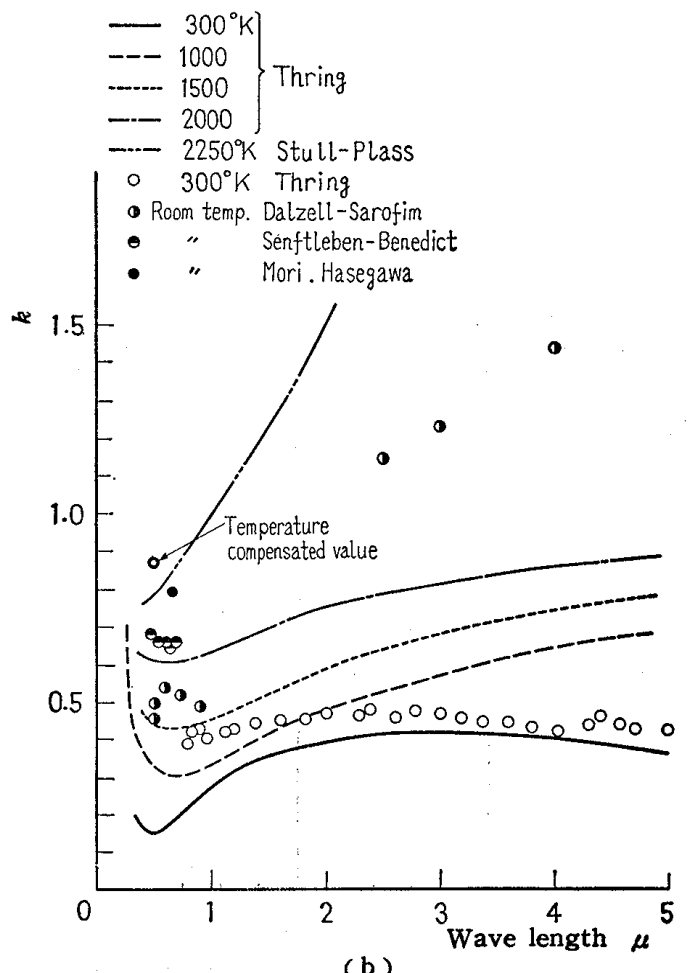

Fig. 9 Complex refractive index and poor agreement is seen between this value and that obtained by use of a transmitted light. It is considered to be due to the variation of physical property of soot of $m$ caused by an increase in temperature. Namely, according to Thring et al. ${ }^{(8)}$, $n$ becomes smaller and $k$ bigger with an increase in soot temperature in the visible region as shown in Fig. 9 (a) and (b). After the temperature is corrected in the said way, a curve for a uniform diameter in Fig. 3 is changed to one illustrated in Fig. 10, where $T_{1}$ is a temperature without an addition of oxygen and $T_{2}$ is that with oxygen introduced, then $T_{2}>T_{1}$. The diameter $D_{T 2}$ at temperature $T_{2}$ is smaller than $D_{T_{1}}$ at $T_{1}$. Values of $D_{32}$ at $T_{1}$ and $T_{2}$, of which the physical properties are corrected, nearly agree with each other. It is beyond the accuracy of this study to make it clear if a value of $D_{32}$ actually varies when oxygen is introduced. It is expected that values of $D_{32}$ obtained from a light-scattering technique and an attenuation method agree with each other by use of a suitable value of $m$ when oxygen is added. A value of $m$ at high temperatures is adopted as:

$$
m=1.73-0.87 i
$$

Values of $D_{32}$ obtained after the correction of Fig. 8 (b) are shown in Fig. 11. They agree fairly well with those without an addition of oxygen. A corrected value varies according to the temperatures; that is, it varies according to the amount of added oxygen. In this study, as the exact measurements on a flame temperature could not be made at high temperatures, it was difficult to go so far as to obtain the variation of $m$ in a wide range of

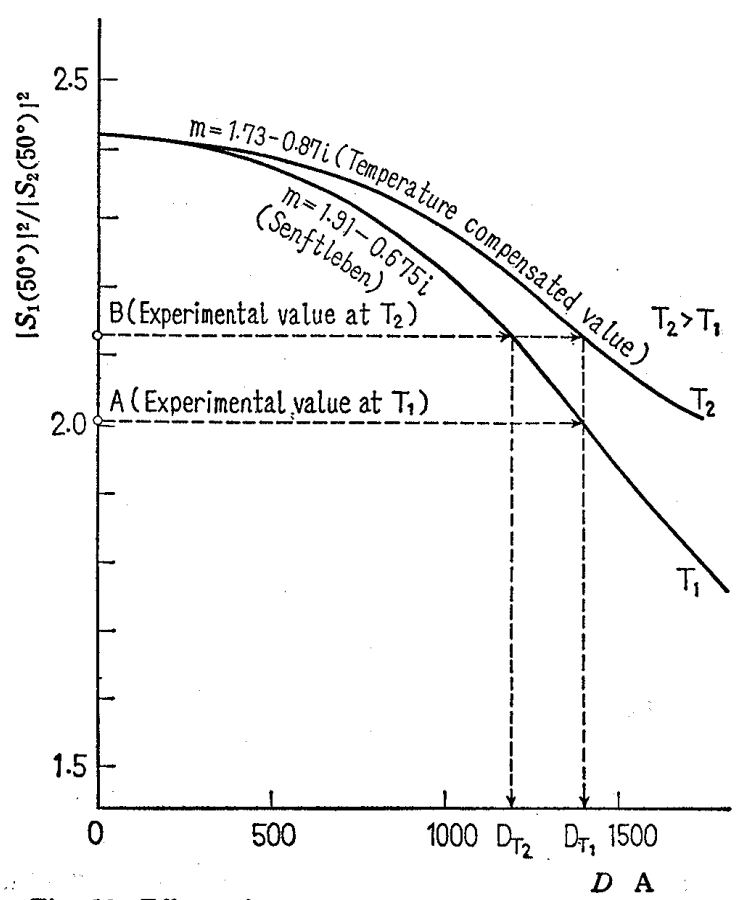

Fig. 10 Effect of temperature on $\left|S_{1}\left(50^{\circ}\right)\right|^{2} /\left.1 S_{2}\left(50^{\circ}\right)\right|^{2}$ 
temperatures. Values of $n$ and $k$ in this study are illustrated in Fig. 9(a) and (b). In addition, the values of $n$ and $k$ obtained by Stull-Plass ${ }^{(14)}$ for carbon at $2250^{\circ} \mathrm{K}$, by Dalzall-Sarofim (11) for soot, by Mori et al. ${ }^{(9)}$ for carbon at room temperature, and those at room temperature by SenftlebenBenedict ${ }^{(7)}$ for carbon are also shown. The temperature-corrected values of $n$ and $k$ used in this study are close to those by Stull-Plass ${ }^{(14)}$ for carbon at $2250^{\circ} \mathrm{K}$. The values of $n$ and $k$ by DalzallSarofim for soot are smaller than those for carbon, because soot used in their experiments is not pure carbon, but $\mathrm{H} / \mathrm{C}$ is $1 / 5 \sim 1 / 15$. It may be, therefore, considered that the number of conduction electrons in soot is fewer than that in carbon; then, the values of $n$ and $k$ become smaller. To make the temperature corrections of transmittency, values of $m$ at each wavelength used in the measurement of transmittency have to be corrected. Therefore, in this study, this point will not be further mentioned because of its complexity.

(b) Variation of $C_{v}$ :

The variation of $C_{v}$ obtained from a transmit.

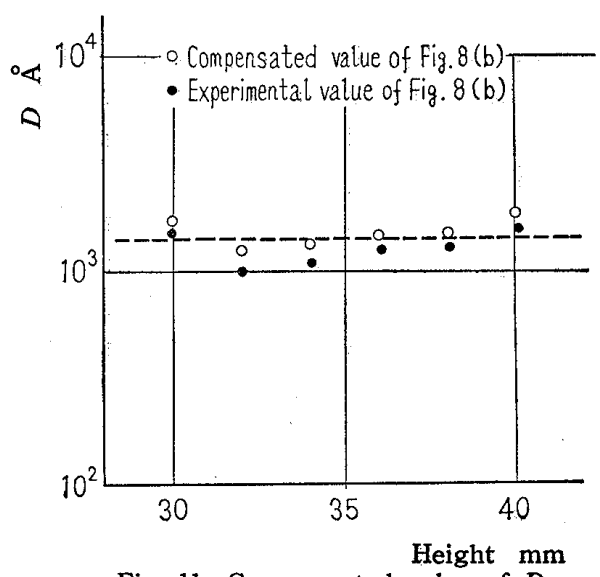

Fig. 11 Compensated value of $D$



Fig. 12 Soot particles concentration of propane diffusion flame (maximum flame height $50 \mathrm{~mm}$ ). tency is indicated in Fig. 12. $C_{v}$ increases with an increase in the flow rate of oxygen. With an increase in the flow rate of oxygen, partial pressure of oxygen becomes higher, and the flame temperature rises. Therefore, when the oxygen lean gas fuel is remarkably preheated, the dehydrogen-reaction of hydrocarbon, which is regarded as one of the mechanisms of the soot generating reaction, is considered to be promoted. As a result, $C_{v}$, the volumetric concentration of soot particles per unit volume of flame, is supposed to become larger.

However, $C_{v}$ stops increasing when partial pressure of oxygen reaches a certain point. It is considered that the concentration gradient of oxygen becomes steeper near the flame boundary, and a diffusion velocity of oxygen into a flame is gained, and combustion of generated soot particles is promoted, but we can not experimentally verify any further.

$C_{v}$ decreases in the direction of the flame height as shown in Fig. 12. However, from Fig. 7, $D_{32}$ remains the same in the direction of the flame height. As the measurements were made on the central part of the flame, the values of $D_{32}$ and $C_{v}$ near that part are given in this study. Because the soot particles burn and disappear near the flame reaction zone, and unburned soot particles in the central part of the flame diffuse toward the flame surface, the concentration of soot particles in the central part is considered to become smaller. Thus, $C_{v}$ decreases in the direction of the flame height.

5.1.2 Experiment in the case of constant flow rate of fuel

(a) Variation of $D_{32}$ :

The comparison between $D_{32}$ at various flow rates of fuel is drawn in Fig. 13 accompanied with respective flame heights. When the flow rate of oxygen is small, that is, the flame temperature is

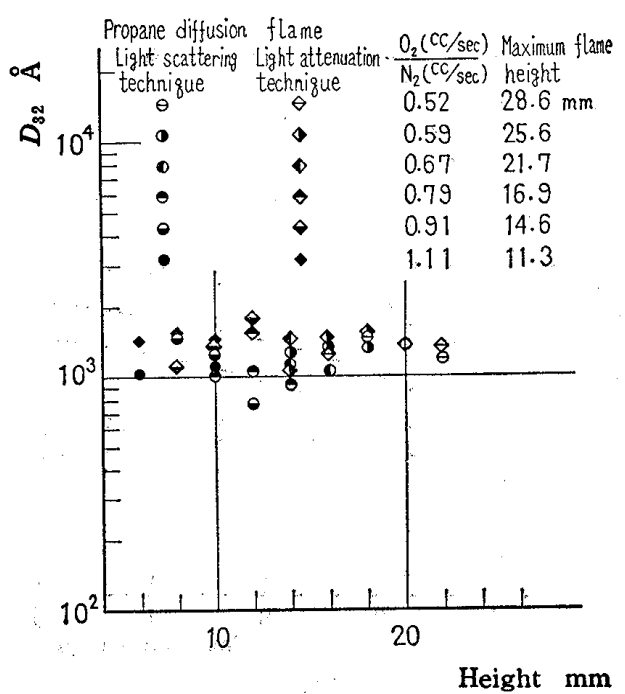

Fig. 13 Effect of oxygen concentration on $D_{32}$ 
not high, good agreement is seen between the values of $D_{32}$ obtained by means of two methods. However, we see poor agreement between them at high flame temperatures, and experimental values are dispersed.

(b) Variation of $C_{n}$ :

The variation of $C_{v}$ obtained by use of a transmitted light is illustrated in Fig. 14. The value of $C_{v}$ becomes larger with an increase in the flow rate of oxygen, which seems to be caused by the same reason as in $5 \cdot 1 \cdot 1$.

$\mathbf{5 . 1 . 3}$ Flame temperature

A distribution of temperatures measured along the axis of a diffusion flame of pure propane of a maximum height of $50 \mathrm{~mm}$ is given in Fig. 15. A dent region was always observed in the temperature distribution of a luminous flame, which was not found in the distribution of a non-luminous flame.

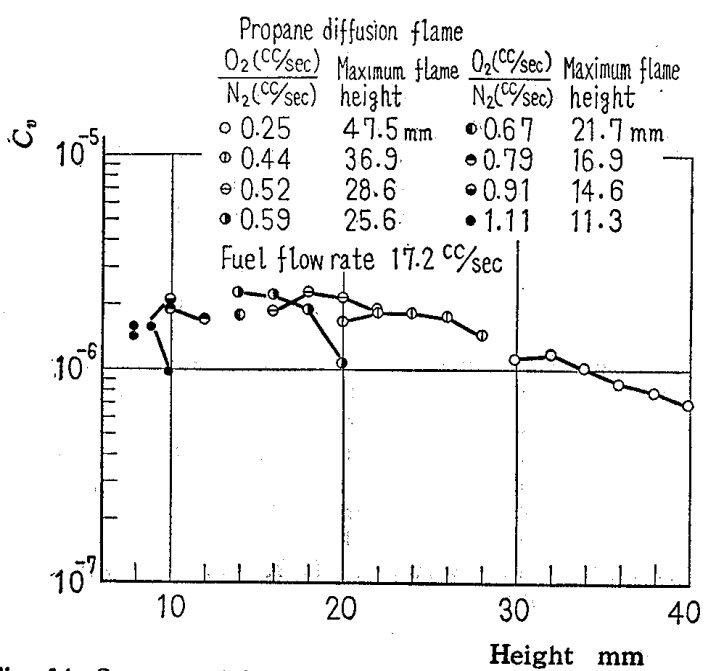

Fig. 14 Soot particles concentration along center line of flame



Fig. 15 Temperature distribution of flame
In this dent region, soot particles instantaneously attached to a thermocouple, and the indicated temperature fell accordingly. A distribution of the actual temperature of this region is not made clear in the experiment. The region coincides with the upper part of the dark zone of unburned gas in the central part of a diffusion flame. The measurement of a scattered light is possible above this region. The pre-soot generating reaction takes place in this region. It is considered that the soot generating reaction is promoted by the insertion of a thermocouple and soot particles attach to its surface. The significance of the reaction is expected to be revealed in a further study. This region was observ. ed to be colored in leaner yellow-red than at the luminous part of the flame. However, a scattered light was not observed in this region, but the decrement of a transmitted light occurred. Therefore, it can be considered that the solid soot particles do not exist in this region, and the luminosity radiated from this region is mainly caused by the chemical reaction, or supposedly by the same kind of the mechanism as observed by Echigo et al. (1)

\subsection{A case in which city gas is used}

Propane was used in the previous experiments. City gas was also tried to take similar measurements. Comparison between values of $D_{32}$ obtained by two methods is made in Fig. 16 and $C_{v}$ is obtained from a transmittency in Fig. 17. Good agreement is seen between the values of $D_{32}$ measured by means of the two methods. Moreover, those for about $1400 \AA$, agree with those obtained in Fig. 7 using propane. However, $C_{v}$ is small by one order. This can be explained from the fact that the fuel with the higher ratio of carbon in molecules generally produce a larger amount of soot particles.

\section{Air premixed flame}

An air premixed flame of pure propane was also measured to compare with the diffusion flame, but no great difference in $D_{32}$ was observed. On

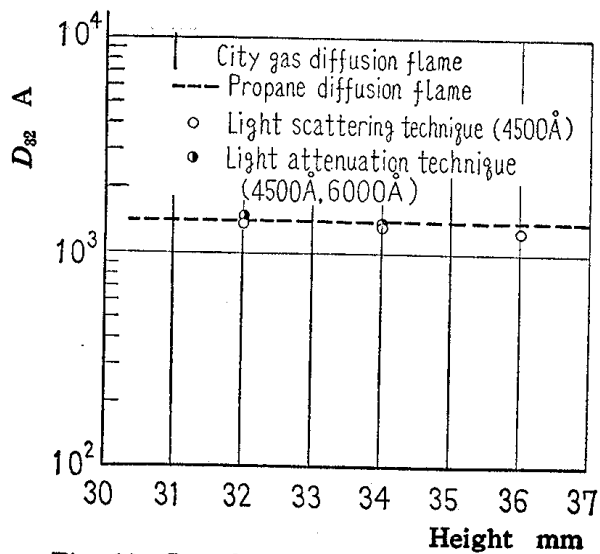

Fig. $16 D_{32}$ of city gas diffusion flame 
the other hand, the value of $C_{v}$ obtained by use of a. transmitted light decreases with an increase in the amount of air and the luminosity of flame decreases, because fuel and oxygen are well mixed and enough oxygen to react with the fuel exists in the unburned region.

\section{Conclusions}

The measurements on the mean diameter and the concentration of soot particles, which act as the main source of radiation of a luminous flame, were carried out by use of scattered and transmitted light intensities of the incident light. After the experimental study on radiation from the luminous flame, we came to the conclusion as follows.

(1) Mie's theory on radiation from a luminous flame is experimentally verified by the use of $D_{32}$, which is adopted as the mean diameter of soot particles in a luminous flame, defined by Eq. (15).

(2) $D_{32}$ hardly varies in the axial direction of a propane diffusion flame, and its value is approximately $1400 \AA$. $C_{v}$ has a tendency to slightly decrease in the direction of the flame height.

(3) When the temperature of a diffusion luminous flame is raised with an increase in oxygen in the surrounding air, it is pointed out that the strict examination of the dependence of physical properties of soot of $n$ and $k$ on a soot temperature

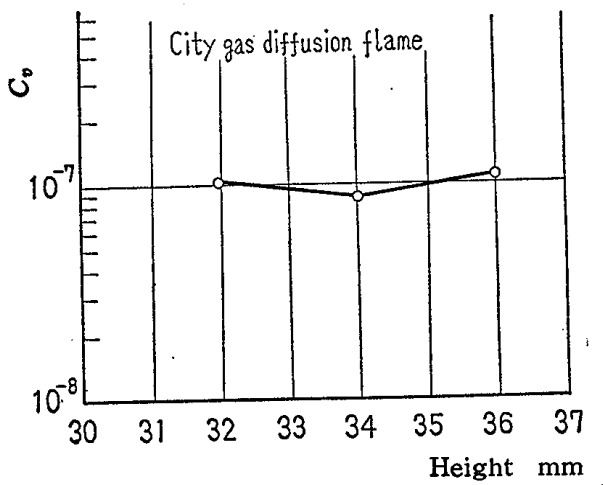

Fig. 17 Soot particles concentration of city gas diffusion flame is necessary to predict soot behavior.

(4) A value of $C_{v}$ increases with an increase in oxygen in the air. However, it stops increasing: after the oxygen ratio reaches a certain value.

(5) Values of $D_{32}$ for a diffusion flame of city gas are nearly the same as those of propane. However, a value of $C_{v}$ is considerably smaller.

(6) There exists a weakly luminous part near upstream of the soot existing region, where. the scattered light intensity is hardly observable, but the transmitted light intensity is decreased.

(7) Concerning an air premixed luminous: flame, values of $D_{32}$ differ little from those for a: diffusion luminous flame. However, the value of $C_{v}$ is considerably smaller.

The authors wish to acknowledge the co-operation of Mr. Kazuo Onda of Electro-technical Laboratory in carrying out this study.

\section{References}

(1) R. Echigo et al.: Trans. Japan Soc. Mech. Engrs., Vol. 32, No. 237 (1966-5), p. 743.

(2) M. Kunugi et al.: Ann. Rept. Asahi Glass Found. for Contribution to Ind. Technol., Vol. 10 (1964).

(3) S. Yagi.: Japan Jour. Chem. Ind., Vol. 2, No. 40 (19372), p. 90 .

(4) T. Sato and R. Matsumoto: Int. Dev. in Heat Transfer, Pt. IV, Sec. B, (1961 62), p. 804, Int. Heat Transfer Conf. ASME.

(5) G. Mie: Ann. Physik, Bd. IV, Nr. 25 (1908), S. 377.

(6) H.C. Van De Hulst: Light Scattering by Small Particles, (1962), John Wiley and Sons.

(7) H. Senftleven and Benedict: Ann. Physik, Bd. IV, Nr. 54 (1917), S. 65.

(8) C.R. Howarth et al.: Proc. of 3 rd Int. Heat Transfer Conf., Vol. V (1966-8), p. 122.

(9) Y. Mori et al.: Preprint of Third Japan Symp. Heat Transfer, (1966-5), p. 189.

(10) W.H. Dalzall and A.F. Sarofim: Private Communication.

(11) R.A. Dobbins and G.S. Jizmagian: Jour. Opt. Soco.' Amer., Vol. 56, No. 10 (1966-10), p. 1345.

(12) M. Kunugi and H. Jinno: 11th Symp. Int. on Comb.s. (1966), p. 257.

(13) W.D. Erickson et al.: Comb. and Flame, Vol. 8, No. 2 (1964-6), p. 127.

(14) V.R. Stull and G.N. Plass: Jour. Opt. Soc. Amer.w Vol. 50 (1960), p. 121 